

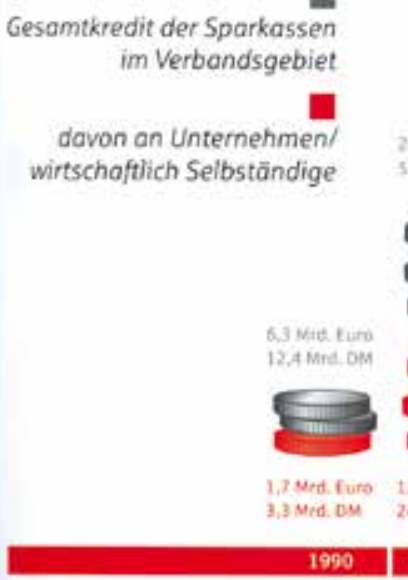

Kreditentwicklung in 25 Jahren. Im Verhältnis betrachtet, sind die Kredite stärker gewachsen als die Einlagen im OSV-Verbandsgebiet. ๑ Ostdeutscher Sparkassenverband

rechts: Der Einheitszinssatz von 3 1/4 Prozent ab 1971 wurde in bereits bestehende Sparkassenbücher gestempelt.

Neue Bücher wiesen einen Eindruck des Zinssatzes, der bis zum Ende der DDR seine Gültigkeit behielt, auf der linken Innenseite aus.

(c) Historisches Archiv des Ostdeutschen Sparkassenverbandes

In der ersten Juliwoche wurden durch die Deutsche Bundesbank rund 4,5 Milliarden D-Mark für Auszahlungen bereitgestellt. Das

Geld kam, wie hier in Grimma auf Lkws, verpackt in Säcken an, wurde entladen und erst einmal in den Büros deponiert. ๑ Sparkasse Muldental
Die den DDR-Sparkassen im Rahmen der Arbeit des Bankensektors zugeteilten Aufgaben bestanden im Wesentlichen in der Hereinnahme von Spareinlagen der Bevölkerung, der Abwicklung des Zahlungsverkehrs und begrenzt der Kreditgewährung. Der zuletzt erreichte Einlagenbestand betrug ca. 130 Milliarden Mark der DDR. Im Kreditbereich vergaben die Sparkassen vor allem private Wohnungsbaukredite und Konsumentenkredite, die streng reglementiert wurden. Konsumentenkredite wurden z. B. nur zum Kauf konkret definierter Waren, die in einem vom Ministerium für Handel und Versorgung herausgegebenen Warenverzeichnis aufgeführt waren, gewährt. Darüber hinaus führten die Sparkassen Konten selbstständiger Handwerker und von Gewerbetreibenden, denen sie ebenfalls in begrenztem Umfang Kredite gewähren durften.

Das Volumen der Wirtschaftskredite der DDRSparkassen erreichte 1989/90 ca. 500 Millionen Mark der DDR. Heute wären das 127,8 Millionen Euro. Ihr gesamtes Kreditvolumen erreichte zuletzt ca. 22 Milliarden Mark der DDR (umgerechnet 5,6 Milliarden Euro). 86 Prozent davon waren für private Wohnungsbaukredite ausgereicht worden.

Heute beträgt der Kreditbestand allein der Sparkassen in Sachsen 22,3 Milliarden Euro, 11,6 Milliarden Euro davon sind Wirtschaftskredite.

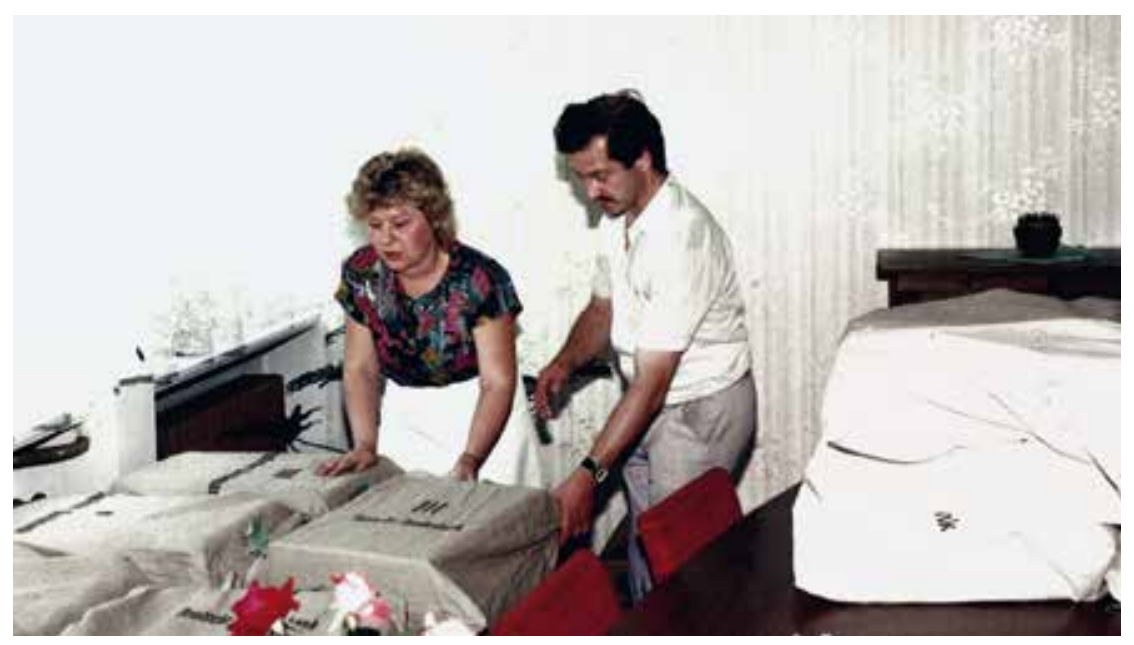

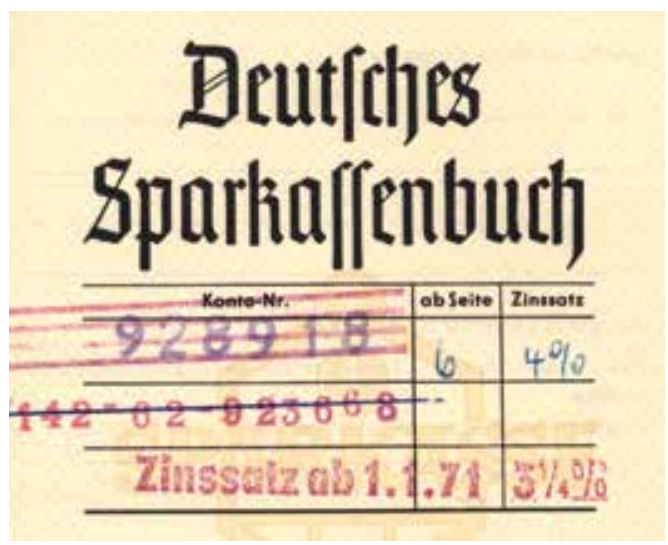

Auch zwischen den angebotenen Sparprodukten für die Kunden gab es zwischen Ost- und WestSparkassen zur Zeit der Herstellung der deutschen Einheit Unterschiede. Die Förderung des Sparens beschränkte sich in der DDR seit 1971 auf ein reduziertes Angebot von Spareinlagen in Form von Buchsparkonten und Spargirokonten mit zentral festgelegter Verzinsung. Andere Sparformen existierten nicht mehr.

Die andere Geschäftspraxis von DDR-Sparkassen war eine logische Folge des existierenden Wirtschafts- und Gesellschaftsmodells sowie seiner strukturellen Erfordernisse, beeinflusst von ideologisch begründeten Auffassungen über die Rolle des Kreditwesens in der sozialistischen Volkswirtschaft.

Die Wirtschafts-, Währungs-, und Sozialunion zwischen der Bundesrepublik Deutschland und der DDR am 1. Juli 1990 sowie zeitversetzt der Beitritt der DDR zur Bundesrepublik Deutschland am 3. Oktober 1990 bedeutete daher für die ostdeutschen Sparkassen und für ihre Beschäftigten einen gewaltigen Umbruch.

Wie auf dem Gebiet der Wirtschaft insgesamt, so war auch im Sparkassensektor die Herstellung der deutschen Einheit keine Vereinigung gleicher Strukturen, sondern der Sprung von einer zentralistisch organisierten Planwirtschaft sozialistischer Prägung in eine dezentrale, kapitalistische Marktwirtschaft mit individueller Unternehmensverantwortung und Wettbewerb. Hinzu kam, dass die Produktvielfalt in jedem Bereich beachtlich zunahm. Dies alles galt es zu meistern, sowohl technisch, als auch in den Köpfen der Beschäftigten.

So gut wie alles im neuen System war anders: die Eigentumsverhältnisse, die Ziele des Wirtschaftens, die Verantwortungsketten, die Entscheidungsprozesse, die Einbindung in die Arbeitsteilung, die Rechtsgrundlagen, die Technik usw. Selbst was gleiche Bezeichnungen trug, musste nicht zwingend gleichen Inhalt haben. So waren Kredite an große DDR-Betriebe vom Wesen her etwas anderes als Kredite an Betriebe in der Bundesrepublik Deutschland. Wirtschaftskredite hatten in der DDR die Funktion von Buchungsposten in der Plan- und Zahlungserfüllung. Die Kreditgewährung diente nicht zuletzt der Steuerung und Kontrolle einzelner Bereiche der Volkswirtschaft durch die Zentrale. 
Die Kreditfinanzierung von DDR-Betrieben war auch die gewollte Folge der Politik der Abführung beachtlicher Teile der Unternehmensgewinne an den zentralen Staatshaushalt.

Die Umstellung auf marktwirtschaftliche Gegebenheiten und die Vorbereitung der Währungsunion musste zudem in einer extrem kurzen Zeitspanne bewältigt werden. Nachdem Bundeskanzler Helmut Kohl am 6. Februar 1990 überraschend eine Währungsunion vorgeschlagen hatte, hatten die Sparkassen vom 7. Februar bis zum 30. Juni 1990 Zeit, um dies vorzubereiten. Ganze vier Monate, drei Wochen und zwei Tage, um sich in die Lage zu bringen, für über 90 Prozent der DDR-Bürger die Währungsumstellung vorzunehmen und ihnen die breite Palette an Finanzdienstleistungen anzubieten, die im Westen Deutschlands zum Standard gehörten. Zum Vergleich: Für die Vorbereitung auf die spätere Einführung des Euro hatte die Kreditwirtschaft mehrere Jahre Zeit.

Trotz der Zeitknappheit und der Zusatzaufgabe Währungsunion gelang der Übergang der DDRSparkassen in das westdeutsche Wirtschafts- und Rechtssystem sehr gut. Sie gehören zu der Minderheit ostdeutscher Unternehmen, die nicht nur weiterexistierten, sondern die an Kraft gewannen und die ihre Marktführerschaft mit Erfolg verteidigen konnten. Dies ist dem entschlossenen Handeln aller Akteure, von den Trägern und der Führungsebene der Sparkassen, bis zur Belegschaft und den Partnern in der Sparkassenorganisation in Ost und West zu verdanken. Positiv wirkte sich aus, dass die Sparkassen flächendeckend präsent geblieben sind und damit in hohem Maße kundennah. Im Ergebnis zeigten die Sparkassenkunden ihrerseits eine sehr hohe Treue, auch gerade in der Anfangszeit, in der es zu einem großen Andrang bei den Sparkassen kam und sie deutlich längere Wartezeiten als Kunden der neu auf dem Markt in Erscheinung tretenden Großbanken hinnehmen mussten. Gab es bis 1989 kaum Kontakte zwischen Sparkassen der Bundesrepublik und der DDR, so änderte sich dies schlagartig nach der Öffnung der Grenze im November 1989. Bereits nach wenigen Wochen kam es in den grenznahen Gebieten zu Gesprächen zwischen Repräsentanten von Ost- und WestSparkassen. Ziel war zunächst das Kennenlernen der anderen Seite, später folgte die Kooperation auf Basis konkreter Hilfsangebote und -anfragen. Dabei ging es Schlag auf Schlag. Vertreter der Abteilung Sparkassen der Staatsbank der DDR und des Deutschen Sparkassen- und Giroverbandes trafen sich Ende Dezember 1989 zunächst noch inoffiziell, aber bereits am 2. Januar 1990 gab es dann ein erstes offizielles Gespräch. Zur Überraschung der westdeutschen Teilnehmer waren die Überlegungen in der Abteilung Sparkassen bereits relativ ausgereift und umfassten Ansätze für die Neugestaltung des Sparkassenwesens in der DDR. Dazu zählte sowohl ein neues Sparkassenrecht als auch die die Absicht der Schaffung eines eigenen, von der Staatsbank der DDR unabhängigen Sparkassenverbandes. Dieser wurde dann auch 1990

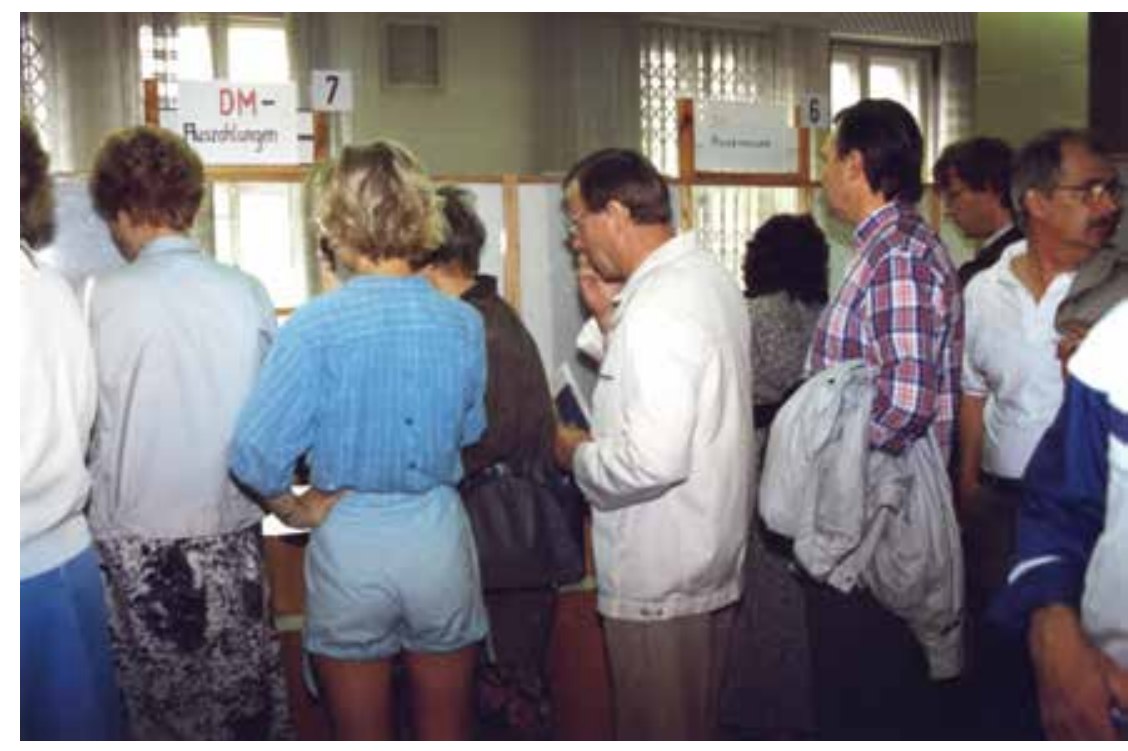

per Beschluss der Modrow-Regierung gegründet. Schon am 20. September 1990 wurde ein außerordentlicher Verbandstag einberufen, um den politischen Realitäten folgend (beschlossenes Ende der DDR, kommende staatliche Einheit Deutschlands und bevorstehende Gründung der ostdeutschen Bundesländer) Rechnung zu tragen und den Verband entsprechend den Gegebenheiten in der Bundesrepublik Deutschland umzubenennen, d. h. die Ländernamen im Verbandsnamen zu verankern (Sparkassen- und Giroverband für die Sparkassen der Länder Brandenburg, Mecklenburg-Vorpommern, Sachsen, Sachsen-Anhalt und Thüringen; Ostdeutscher Sparkassen- und Giroverband). Später folgten noch zwei Namensänderungen, die letzte 2005, die zur heutigen Kurzform „Ostdeutscher Sparkassenverband“ führten.

In Folge der staatlichen Einheit und der Gründung der Länder am 3. Oktober 1990 wurde auch die Frage aufgeworfen, ob ein einheitlicher ostdeutscher Sparkassenverband bestehen bleiben sollte. Alternativ wurde die Gründung von SparkassenLänderverbänden oder der Anschluss an beste-

Am Sonntag, dem 1. Juli 1990, standen die Bürger der DDR nach dem „neuen Geld" an. Der Andrang war überall groß. Zunächst konnten 2.000 D-Mark bar, ab dem 9. Juli Beträge in unbegrenzter Höhe vom Konto abgehoben werden.

( ) Historisches Archiv des

Ostdeutschen Sparkassenverbandes

Am 20. März 1990

erfolgte die Gründung des Sparkassenverbandes der DDR in Strausberg. Alle Leiter der 196 ostdeutschen Sparkassen waren anwesend. Sie verabschiedeten die erste Verbandssatzung und wählten den ersten Präsidenten, Rainer Voigt (links im Bild). Foto: Klaus-Peter Matte

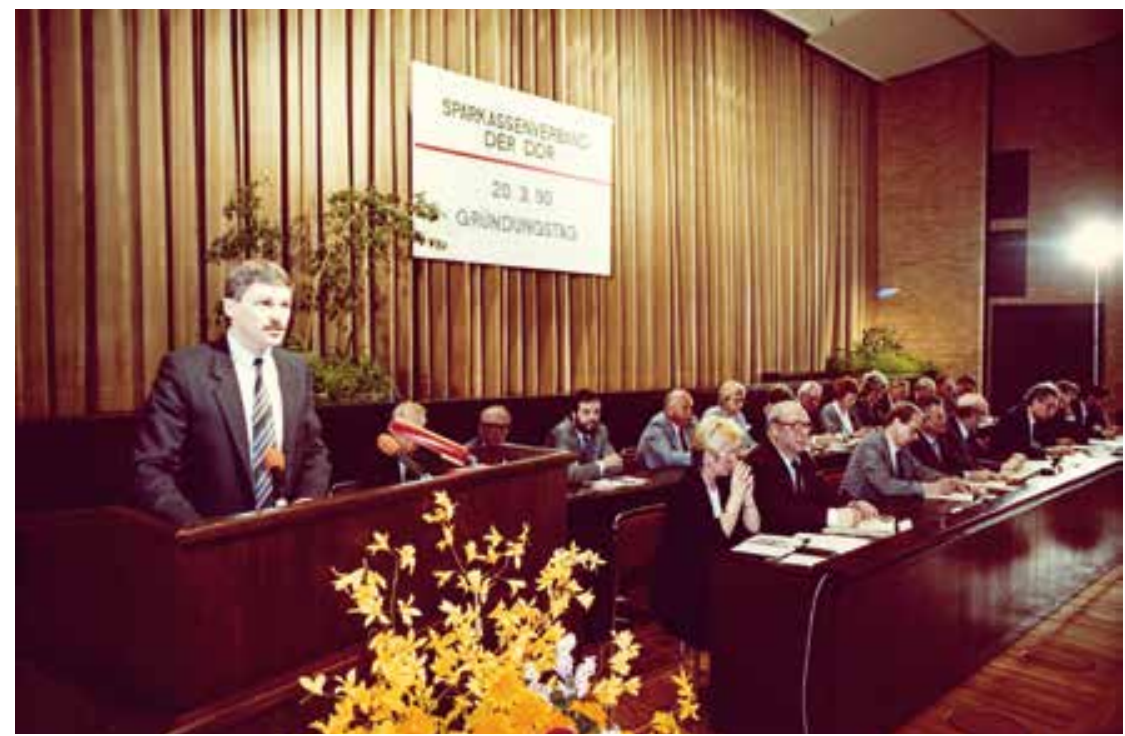


Am 29. Mai 1990 übergab der Präsident des Deutschen Sparkassen- und Giroverbandes, Helmut Geiger (links im Bild),

in Berlin den ersten von

1.000 Personalcomputern

an den Präsidenten des

Sparkassenverbandes der DDR, Rainer Voigt.

๑ Archiv des Deutschen Sparkassen- und Giroverbandes

Stellenanzeige in der Deutschen Sparkassenzeitung, 18. September 1990

๑ Deutsche Sparkassenzeitung

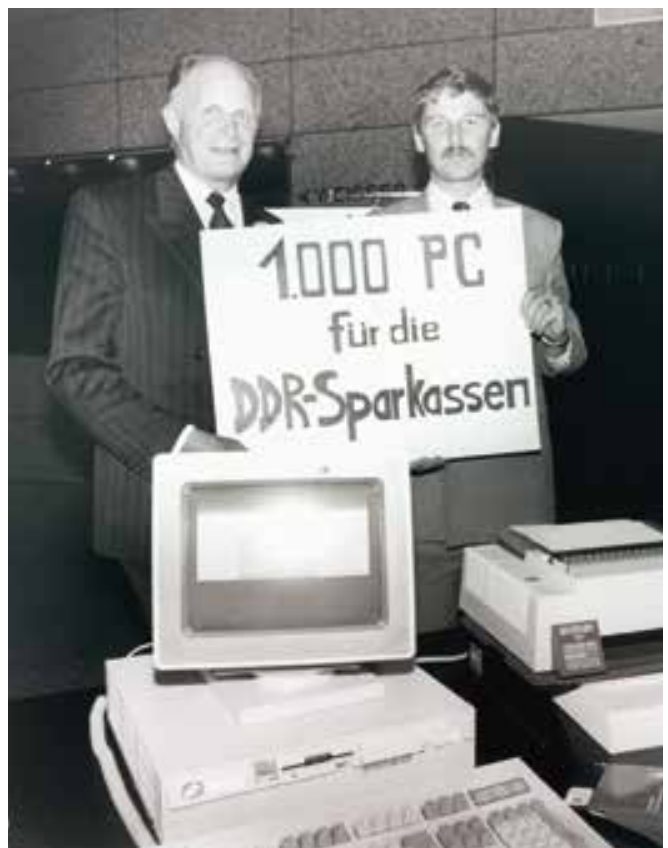

hende westdeutsche Sparkassenverbände diskutiert. Es sollte bis Februar 1992 dauern, bis die Länder Brandenburg, Mecklenburg-Vorpommern, der Freistaat Sachsen und Sachsen-Anhalt sich auf einen gemeinsamen Sparkassenverband verständigt hatten und darüber einen Staatsvertrag abschlossen. Sie sahen es als vorteilhaft an, die Kräfte auf diesem Gebiet zu bündeln, nicht zuletzt, da die Sparkassen alle die gleichen Ausgangsbedingungen und Interessen hatten und auch dem gleichen Wandlungsprozess unterlagen. Im Freistaat Thüringen verlief diese Diskussion auf landes- und kommunalpolitischer Ebene anders. Im Ergebnis
Die

\section{Kreissparkasse Meißen}

schreibt hiermit für ihr Unternehmen mit einem Geschäftsvolumen von $850 \mathrm{Mil}$. lionen Mark, 21 Geschüftsstellen und 150 hauptamtlichen Beschäftigten die Anstellung von

\section{Vorständen}

darunter

\section{Vorsitzender}

Gesucht werden: Führungskräfte mit Durchsetzungsvermògen und Bereitschaft zu überdurchschnittlichem Engagement,

- analytischem Denkvermögen und Urteilskraft,

- mehrjăhrige Berufserfahrung.

Den erfolgreichen AbschluB einer einschlägigen Berufsausbildung sowie eine wirtschaftswissenschaftliche Ausbildung oder ein vergleichbares Studium setzen wir voraus.

Wir bieten: Eine Anstellung mindestens für 5 Jahre, It. $\$ 15$ SpKG, auf Grundlage eines Dienstvertrages sowie moderne Lebensbedingungen im historischen, zu rekonstruierenden MeiBen.

Bewerbungen bitten wir bis zum 25. September 1990 an die

Vorsitzende des Verwaltungsrates, Frau Koch, Landratsamt O-8250 Meißen, zu richten. wurde dort entschieden, sich dem Sparkassenund Giroverband Hessens anzuschließen bzw. per Staatsvertrag einen gemeinsamen Verband mit Hessen zu bilden, was dann umgesetzt wurde. 1990 bestand Einvernehmen darin, dass die Sparkassen - nach der für notwendig erachteten Wirtschaftsreform - ihre Aufgabe nur als öffentlichrechtliche Institute in kommunaler Trägerschaft, damals noch in Form der Gewährträgerhaftung, erfüllen könnten. Das hieß, dass nach knapp vierzig Jahren als volkseigene Kreditinstitute die Sparkassen wieder als „echte“, das heißt kommunal getragene und gebundene, auf das Träger-Territorium ausgerichtete und demokratisch kontrollierte Kreditinstitute tätig sein sollten und wollten. Es galt, möglichst rasch passfähige unternehmerische Strukturen und ein den westdeutschen Sparkassen vergleichbares Dienstleistungsangebot für Privat-, Geschäfts- und öffentliche Kunden herzustellen. All dies ging nicht ohne Hilfestellung, die auch breit von den westdeutschen Sparkassen gegeben wurde. Vorteilhaft war die vom Deutschen Sparkassen- und Giroverbandes (DSGV) ins Leben gerufene Solidaraktion für die rasche Eingliederung der DDR-Sparkassen in das gewachsene bundesdeutsche Sparkassensystem. Regionale Sparkassen- und Giroverbände der Bundesrepublik übernahmen, abgestimmt mit dem jungen Sparkassenverband der DDR, Partnerschaften für die Sparkassen in den fünfzehn Bezirken der DDR. Innerhalb kürzester Zeit waren jeder der damals 196 Sparkassen eine oder mehrere westdeutsche Partnersparkassen zugeordnet. In Sachsen kamen diese aus naheliegenden Gründen aus Süddeutschland.

Diese Partnerschaften hatten zum Ziel, die Organisation und Durchführung von Schulungsmaßnahmen zu unterstützen sowie personelle und materielle Hilfe zu gewähren. Die materielle Hilfe orientierte sich am dringenden, kurzfristigen Bedarf. Sie reichte von Büromaterial bis zu Frankiermaschinen und Kraftfahrzeugen. So umfasste die offizielle Wunschliste der DDR-Sparkassen im Februar 1990 z. B. 2.638 Kopiergeräte, 2.239 Tischrechner, 1.440 Schreibmaschinentische 2.528 Schreibtische, 368 Additionsmaschinen, 191 Geldzählmaschinen, 194 Frankiermaschinen aber auch Büroklammern, Briefumschläge, Stempelfarbe, Durchschlagpapier u.a.m. Außerdem wurden rund 1.000 Computer gespendet, die eine wichtige technische Grundlage für die Vorbereitung der Währungsumstellung zur Jahresmitte 1990 darstellten.

Die ersten Schulungen begannen bereits vor der Wirtschafts- und Währungsunion. Auch praktisch unterstützten die westdeutschen Sparkassen ihre ostdeutschen Kollegen vor Ort, insbesondere bei der Bearbeitung von Kreditanträgen von Existenzgründern und mittelständischen Betrieben. Zeitgleich fuhren ostdeutsche Sparkassen-Beschäftigte Richtung Westen, um ihren Kollegen dort über die Schultern zu schauen.

Schnell wurde damit begonnen, typische Sparkassen-Verbundunternehmen aufzubauen, dar- 
unter eine Bausparkasse und ein Rechenzentrum. Hintergrund war, dass Sparkassen einen großen Teil ihrer Produktvielfalt, Flexibilität, Effizienz und Stärke aus dem Unternehmensverbund der Sparkassen-Finanzgruppe schöpfen. Der Verbund ermöglicht es ihnen, ihren Kunden, jederzeit, kostengünstig eine breite Dienstleistungs- und Produktpalette zu marktfähigen Preisen anzubieten. Zum Verbund gehören außerdem die DekaBank, Leasing- und Factoringgesellschaften, Beratungsunternehmen, Wirtschaftsprüfungseinheiten, Landesbanken, ein Online-Broker, Versicherer, ein Verlagshaus, regionale Sparkassenverbände mit deren Prüfungsstellen und Akademien, ein Spitzenverband und andere mehr. Insgesamt rund $600 \mathrm{Un}$ ternehmen. Im Rahmen des Ostdeutschen Sparkassenverbandes wurden eine Prüfungsstelle und eine Sparkassenakademie aufgebaut.

Über die Motive der westdeutschen Hilfe zur Selbsthilfe wurde viel spekuliert. Die Antwort auf die Frage ist, dass für die Unterstützung der ostdeutschen Sparkassen sowohl gesamtwirtschaftliche, bankpolitische als auch grundsätzliche geschäftspolitische Überlegungen sprachen. Die Umwälzung im Osten war nur mit einem leistungsfähigen, hauptsächlich dezentral organisierten und dort auch verantworteten kreditwirtschaftlichen Angebot zu bewältigen. Den westdeutschen Sparkassen war bewusst, dass ihre ostdeutschen Schwesterinstitute in kurzer Zeit leistungsfähige Strukturen entwickeln mussten, wenn sie denn im Wettbewerb mit den privaten Großbanken bestehen sollten. Das Interesse der westdeutschen Sparkassen daran war gegeben, da anzunehmen war, dass eine negative Sparkassen-Entwicklung im Osten mittelfristig auch nachteilige Rückwirkungen auf die Marktstellung der Sparkassen im Westen gehabt hätte. Die Hilfe für die ostdeutschen Sparkassen war daher zugleich auch ein präventiver Beitrag zur Vermeidung negativer Auswirkungen auf die gesamte deutsche Sparkassenorganisation.

Die Investitionskosten, die die ostdeutschen Sparkassen zu schultern hatten, waren immens. Allein für die Umstellung des Datenverarbeitungssystems waren rund 500 Millionen DM erforderlich. Parallel mussten sämtliche Geschäftsabläufe an neue Standards angepasst werden. Sie hatten den Regelungen der Aufsicht, den rechtlichen, betriebswirtschaftlichen und nicht zuletzt personellen Erfordernissen der neuen Zeit zu entsprechen. Allein für die Bewältigung der Währungsumstellung wurde ein zusätzlicher Personalbedarf von 11.000 Personen ermittelt, der nur durch Aushilfskräfte gedeckt werden konnte. Somit bestand eine weitere Herausforderung darin, möglichst schnell zusätzliche Mitarbeiterinnen und Mitarbeiter zu finden und diese sowie das vorhandene Personal zu qualifizieren.

Ebenfalls mussten Regelungen für die Vorstandsebene der ostdeutschen Sparkassen gefunden werden. Bewährt hat sich die Entscheidung, möglichst in vielen Sparkassen dabei auf ein „gemischtes Doppel“ zu setzen: Danach bildeten je-

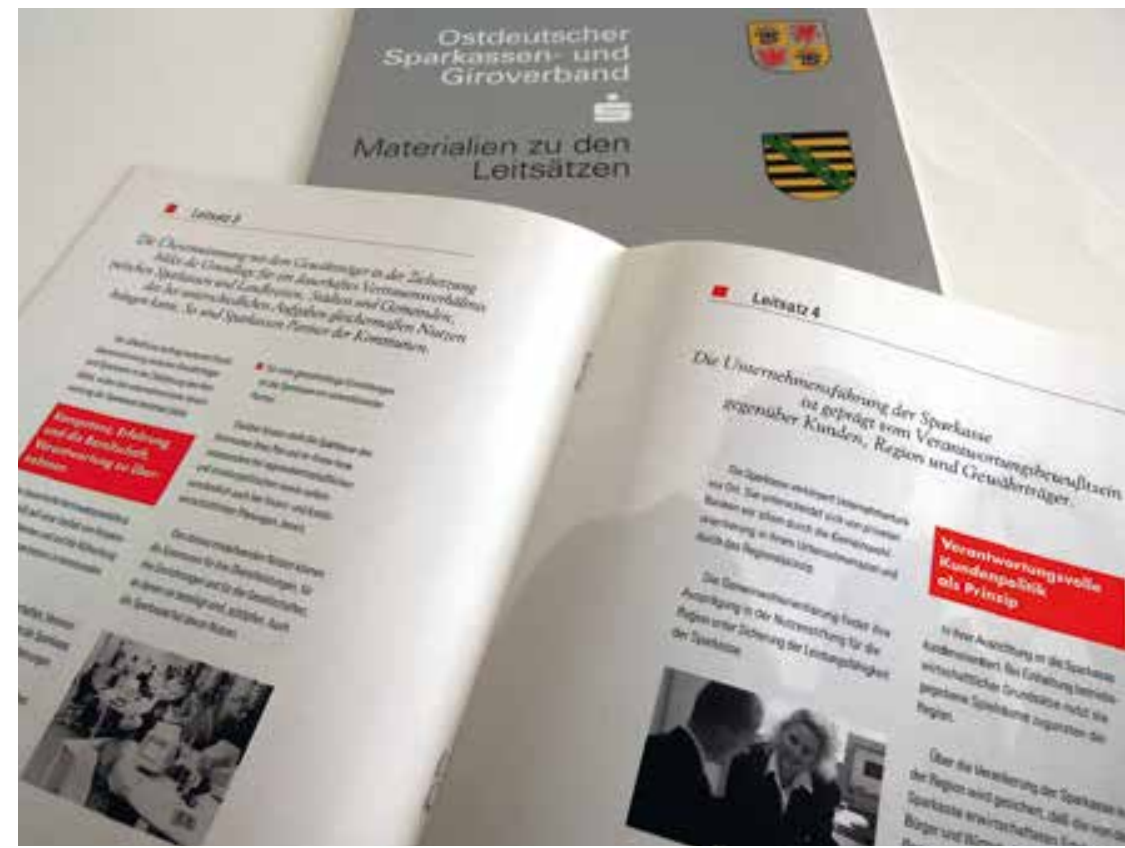

weils ostdeutsche und westdeutsche Vorstände ein Vorstandsgespann. So konnten sowohl die regionale Markt- und Menschenkenntnis als auch das marktwirtschaftliche westdeutsche Know-how für den Umbau der Sparkassen genutzt werden. Beides war für den Erfolg im Wettbewerb unverzichtbar. Viele der westdeutschen Kollegen lebten sich hierzulande so gut ein, dass sie dauerhaft blieben. Bis Mitte der 1990er Jahre hatten sich die ostdeutschen Sparkassen im Markt sehr gut etabliert und waren Marktführer nach Girokonten und Einlagen sowie Krediten an wirtschaftlich Selbstständige und Privatpersonen.

Auf der Basis ihrer zugleich erreichten guten betriebswirtschaftlichen Position formulierten sie 1999 in ihren „Rostocker Leitsätzen“ strategische Überlegungen, die wesentliche Kernaussagen zur weiteren Entwicklung im 21. Jahrhundert enthielten. Darin wurde speziell die der Wirtschaft und dem Gemeinwesen dienende und nutzenstiftende

Auf dem 3. Sparkassentag in Rostock 1999 wurden die noch heute gültigen „Rostocker Leitsätze" verabschiedet. - Historisches Archiv des Ostdeutschen Sparkassenverbandes
Sparkassen-Finanzkonzept. Mit den Mitgliedssparkassen wurde 2002 eine ganzheitliche Beratungssystematik für Privatkunden entwickelt, welche die persönlichen Wünsche und Ziele des Kunden konsequent in den Mittelpunkt rückt.

- Historisches Archiv des Ostdeutschen Sparkassenverbandes

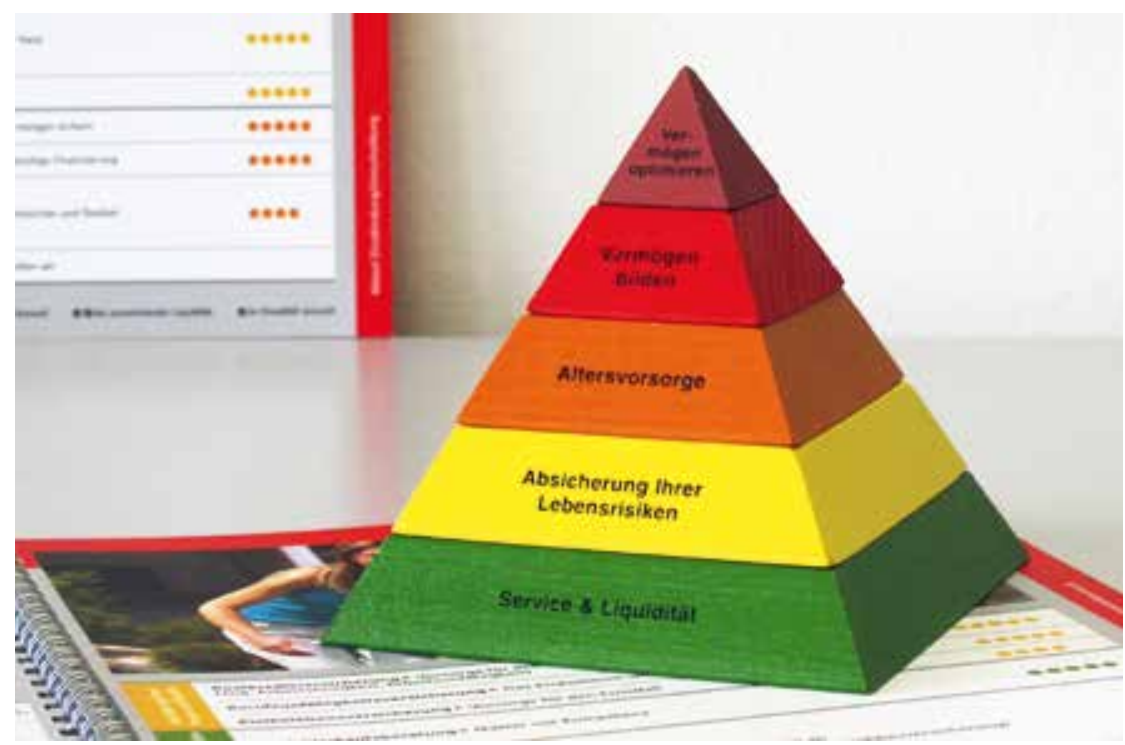


Rolle von Sparkassen sowie ihre feste kommunale Verankerung, ihre Verzahnung mit dem jeweiligen Träger in den Mittelpunkt gestellt. In der damaligen Hoch-Zeit der Shareholder-Value-Ideologie wurde das nicht selten als rückschrittlich belächelt.

Mit Ausbruch der internationalen Finanzmarktkrise ab 2007 zeigte sich, dass die Sparkassen die richtigen Weichenstellungen vorgenommen hatten. $\mathrm{Zu}$ dieser Strategie gehörte, als Voraussetzung der Nutzenstiftung für das jeweilige Trägergebiet, die Sicherung einer starken betriebswirtschaftlichen
Verfassung der Sparkassen, denn nur wirtschaftlich starke Sparkassen können gesellschaftlichen Nutzen stiften. Mittels interner Optimierung von betrieblichen Prozessen und der Bündelung von Aufgaben im Sparkassenverbund sowie des gezielten Ausbaus ihrer auf die Interessen der Kunden und deren Bedürfnisse gesteigerten Vertriebsstärke, gelingt es den ostdeutschen Sparkassen auch weiterhin, dies sicherzustellen.

Seit Mitte des ersten Jahrzehnts des neuen Jahrtausends entwickelten sich die ostdeutschen Sparkassen so gut, dass sie innerhalb der Sparkassenfami-

Finanzgruppe
Ostdeutscher Sparkassenverband

Karte mit 45 Mitgliedssparkassen im Ostdeutschen Sparkassenverband - Ostdeutscher Sparkassenverband

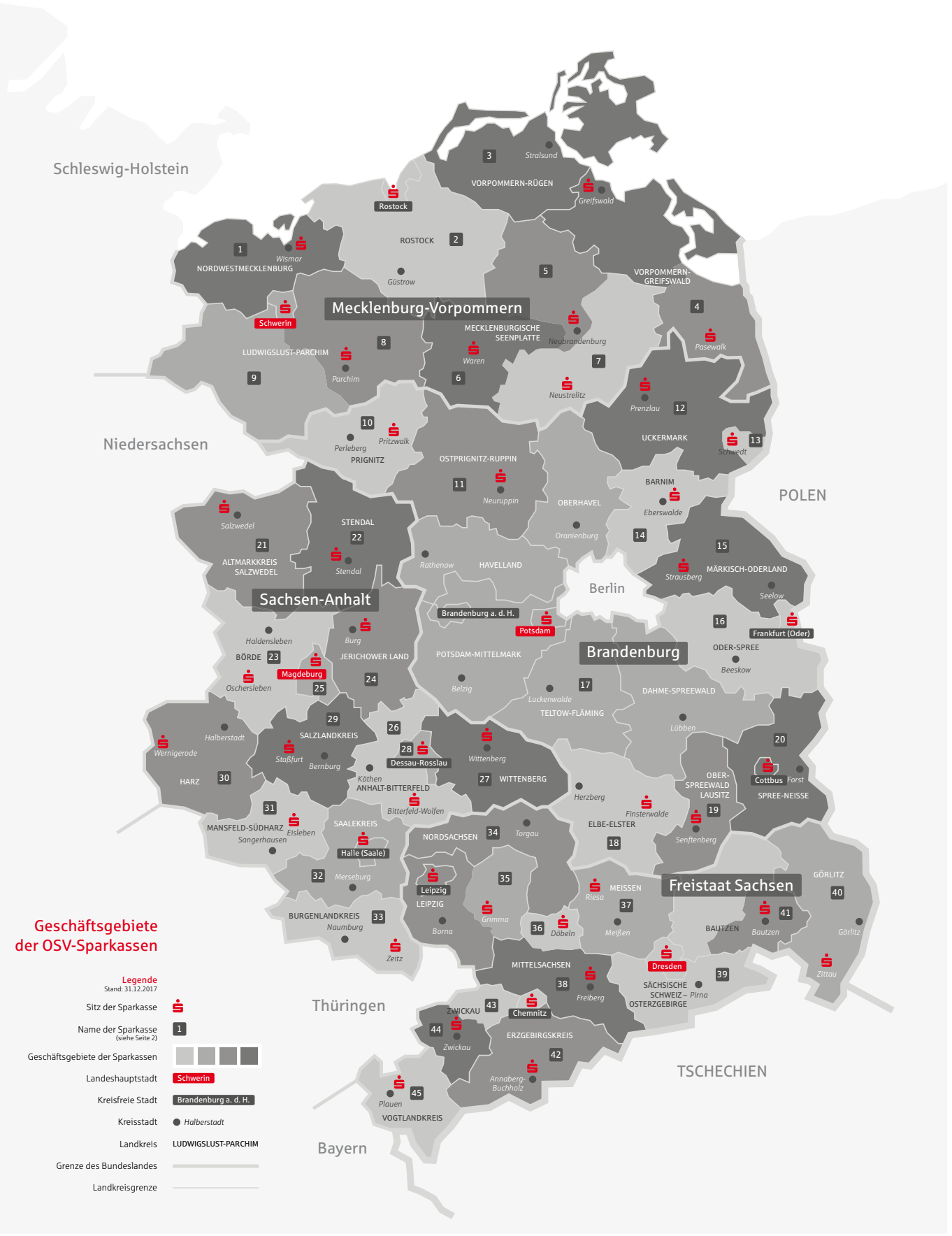


lie - verglichen mit dem Durchschnitt der deutschen Sparkassen - regelmäßig zur bundesweiten Spitzengruppe zählen.

Erfolgreich waren die ostdeutschen Sparkassen auch mit zahlreichen Projekten und Neuerungen. Viele Ergebnisse und Produkte, die im Osten entwickelt wurden, werden heute in der Sparkassengruppe bundesweit eingesetzt. Dazu zählt zum Beispiel das Sparkassen-Finanzkonzept mit seinem Finanz-Check, das in allen deutschen Sparkassen zum StandardBaustein für eine kunden- und bedarfsorientierte Beratung von Privat- und Firmenkunden geworden ist. Es wird sogar von Wettbewerbern kopiert und findet inzwischen auch im Ausland Nachfrage.

Allein in den vier Bundesländern Brandenburg, Mecklenburg-Vorpommern, Sachsen und SachsenAnhalt wirken heute 45 Sparkassen mit rund 1.300 Geschäftsstellen und etwa 20.000 Beschäftigten. Sie stellen 2.800 Geldautomaten für rund 11 Millionen Einwohner zur Verfügung. Rund 30 Prozent aller gewerblichen Finanzierungen werden von ihnen zugesagt, darunter 84 Prozent aller Handwerkskredite.

Nicht zuletzt sind die Sparkassen aus ihrer Tradition heraus dem Gemeinwohl verpflichtet und haben sich zum größten nicht staatlichen Sport- und Kulturförderer entwickelt. Die monetäre Unterstützung regionaler und überregionaler Aktivitäten in den Bereichen Kultur, Sport, Soziales und Jugend beträgt rund 56 Millionen Euro jährlich.

Rückblickend kann festgestellt werden, dass die Integration der DDR-Sparkassen in die große deutsche Sparkassenfamilie ein anhaltendes Erfolgsprojekt war.

Ein Merkmal, das sich gerade in schwierigen Zeiten bewährt hat, ist die Flexibilität der ostdeutschen Sparkassen. Sie haben diese mehrfach unter Beweis gestellt. Zu Beginn der 1990er Jahre bei der Herstellung der Einheit, später im Rahmen von Kreisgebietsreformen bei einer großen Anzahl von Fusionen, danach bei der Euro-Einführung und in der Hochphase der Finanzmarktkrise als Stabilisatoren der Wirtschaft. Parallel dazu haben sie sich immer wieder auf neue Wettbewerber und Marktherausforderungen eingestellt und sind bisher relativ gut mit Niedrigzinsen, demografischem Wandel und die häufig auf sie nicht gut passende Bankenregulierung zurechtgekommen.

Der deutsche Bankenmarkt erlebt seit Jahren einen starken Strukturwandel, der durch einen verschärften Wettbewerb, den Markteintritt von Non-, Near-, New-Banks und Fintechs geprägt ist. Dies berührt zweifelsfrei auch die Arbeits- und Wettbewerbsbedingungen der ostdeutschen Sparkassen. Hinzu kommen neue Entwicklungen und Anforderungen des Marktes, ein zunehmender Margenverfall auf der Ertragsseite, Wirkungen der voranschreitenden Globalisierung und ein starkes Wachstum kapitalmarktgetriebener Geschäftsfelder, was nicht zuletzt durch eine fortschreitende Digitalisierung und Industrialisierung gekennzeichnet ist.

Die Zahl der Banken nimmt kontinuierlich ab, auch die Anzahl der Sparkassen reduzierte sich durch

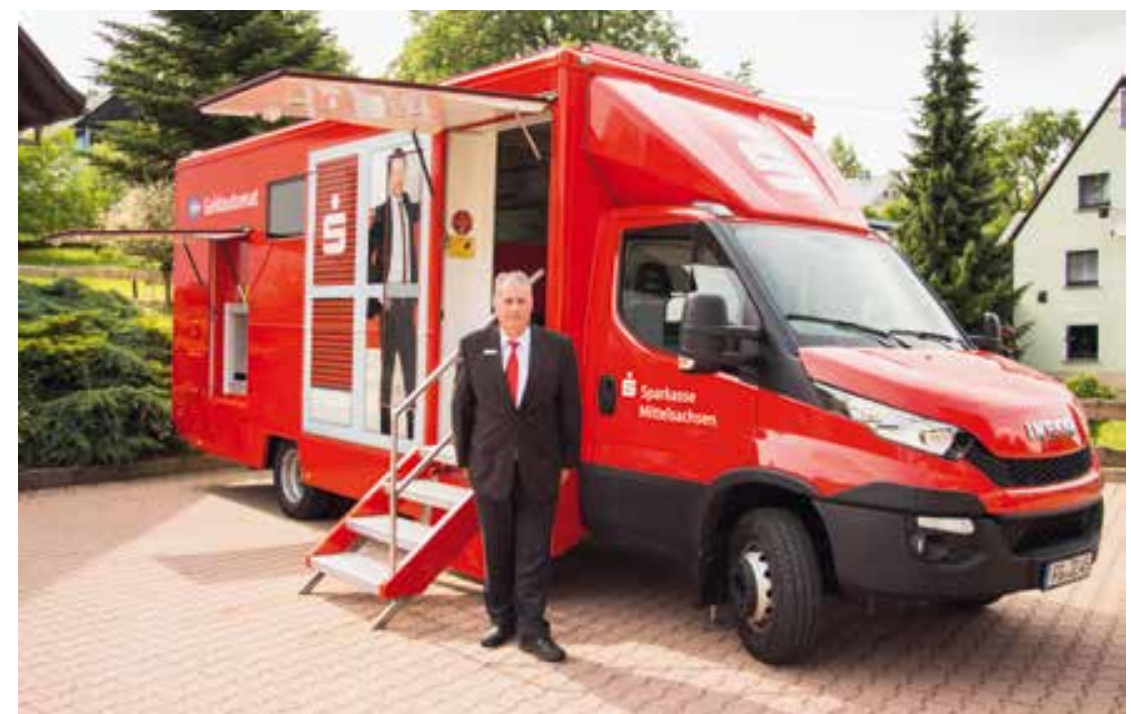

Fusionen von 769 im Jahr 1990 auf 385 im Jahr 2017. Die kreditwirtschaftliche Versorgung aller Landkreise und Städte in Deutschland bleibt dabei als öffentlicher Auftrag der Sparkassen bestehen. Mit Blick in die Zukunft kann festgestellt werden, dass die Sparkassen in Ostdeutschland für ihre Zukunft ausreichend Potenzial haben, um den eingeschlagenen Kurs halten und ausbauen $\mathrm{zu}$ können. Ihre Aufmerksamkeit liegt derzeit vorwiegend in der Weiterentwicklung der betriebswirtschaftlichen Effizienz und der Servicequalitäten der Sparkassen. Einem Vertriebserfolg um jeden Preis erteilen ostdeutsche Sparkassen nach wie vor eine klare Absage. Ziel ist die langfristige und von gegenseitigem Vertrauen sowie Respekt getragene Kundenbeziehung, nicht der schnelle Gewinn.

Der Bevölkerungsrückgang in der Fläche führte dazu, dass die ostdeutschen Sparkassen bereits sehr frühzeitig Lösungsansätze entwickelten, um ihrem Auftrag der flächendeckenden Versorgung und der Nähe gerecht zu werden und dennoch betriebswirtschaftlich sinnvoll zu agieren. Dabei wurden und werden bestehende Strukturen überdacht und ggf. angepasst. Im Zuge sich verändernder Kundenbedürfnisse und der Fortentwicklung moderner Kommunikationstechnologien entstehen komplett neue Formen der Kundenbetreuung, die neben den traditionellen Formen, wie der Geschäftsstelle, zum Gesamtspektrum der Angebote für die Kunden gehören.

Für die ostdeutschen Sparkassen hat sich gezeigt, dass es ihr Vorteil war und ist, ihre Geschäftsausrichtung und ihre Strukturen frühzeitig anzupassen. Sie sind weiterhin gut kapitalisiert, wettbewerbsstark, betriebswirtschaftlich erfolgreich.

Auf diesem Fundament stehend, sehen sie sich für die Zukunft gut gerüstet. Dabei setzen sie auf Grundelemente auf, die dazu führten, dass Sparkassen bereits seit über 200 Jahren erfolgreich arbeiten können: die Nähe zu den kommunalen Trägern, eine enge Kundenbindung, das Vertrauen der Bevölkerung und Flexibilität angesichts sich ändernder Rahmenbedingungen.
Seit über 20 Jahren bietet die Sparkasse Mittelsachsen mit dem Sparkassenbus eine "Mobile Filiale" an und stellt damit den Service rund ums Geld in bevölkerungsarmen Regionen sicher.

๑ Sparkasse Mittelsachsen

\section{Autor}

Wolfram Morales

Leiter des Büros des

Geschäftsführenden Präsidenten

Ostdeutscher

Sparkassenverband

Leipziger Straße 51,

10117 Berlin

wolfram.morales@osv-online.de 